\title{
Pensamento computacional e formação de professores da educação básica: uma revisão da literatura
}

\author{
Claudia da Silva Gomes, IFRS-POA, claug@ufrgs.br, \\ https://orcid.org/0000-0001-9215-9358 \\ Karen Selbach Borges, IFRS-POA, karen.borges@poa.ifrs.edu.br, \\ https://orcid.org/0000-0001-9358-9798 \\ Rodrigo Prestes Machado, IFRS-POA, rodrigo.prestes@poa.ifrs.edu.br, \\ http://orcid.org/0000-0003-0428-6387
}

\begin{abstract}
Resumo: Mundialmente, o Pensamento Computacional vem sendo reconhecido como competência fundamental para todos os sujeitos, sendo introduzido nos currículos escolares desde a educação básica. A partir desse entendimento, este artigo apresenta uma revisão da literatura sobre o CT (acrônimo de Computational Thinking - Pensamento Computacional) para uma reflexão acerca do contexto brasileiro, buscando a identificação e análise sobre a presença, forma e objetivo de seu emprego junto à educação básica. A metodologia adotada possui elementos de um Mapeamento Sistemático, conduzida de forma a buscar respostas para as questões: o CT é abordado na formação de professores para a educação básica? Se afirmativo, de que forma ele é abordado e com que objetivo? A partir da análise das publicações encontradas, foi identificada a presença do CT na educação básica, principalmente em atividades com uso de linguagem de programação, empregada como estratégia didática, no intuito da promoção do desenvolvimento cognitivo, seguido do aspecto atitudinal e de colaboração. Paralelamente, os dados revelam, além de um maior número de experiências formativas com docentes em relação a discentes, uma menor incidência de uso, seja docente ou discente, junto à etapa da educação infantil e anos iniciais do ensino fundamental da educação básica.
\end{abstract}

Palavras-chave: Pensamento Computacional; formação docente; educação básica.

\section{Computational thinking and basic education teacher's training: a review of the literature}

\begin{abstract}
Worldwide, Computational Thinking (CT) has been recognized as a fundamental competence for all subjects, being introduced in school curricula since basic education. Based on this understanding, this paper shows a review of the literature about $\mathrm{CT}$ in the Brazilian context, seeking to identify and analyze the presence, form and purpose, and the application in basic education. The methodology used has elements of a Systematic Mapping in order to answer two questions: is the CT addressed in the training of teachers for basic education? If so, what is the approach and purpose? Through data analysis identified the presence of CT in basic education in order to promote cognitive development, followed by the attitude and collaboration aspects. CT is used mainly as a didactic strategy in activities using programming languages. Also, there was a greater number of training experiences with teachers in relation to students and a lower incidence of explorations with teacher and students along the stage of early childhood education and early years of basic education in basic education.
\end{abstract}

Keywords: Computational Thinking; teacher training; basic education. 


\section{Introdução}

O sistema escolar brasileiro se divide em educação básica e ensino superior, sendo a educação básica estruturada por etapas e modalidades de ensino, as quais abarcam a educação infantil, o ensino fundamental e o ensino médio. Especificamente ao que se refere aos anos iniciais, segundo dados de 2017, do documento Compromisso Nacional pela Educação Básica (BRASIL, 2020), as taxas de insucesso (soma de reprovação e abandono) no sistema escolar brasileiro chegaram a alcançar até $10,5 \%$ ( $3^{\circ}$ ano dos anos iniciais). O mesmo documento destaca a intrínseca relação entre o insucesso educacional e a desigualdade social, elencando, sinteticamente, como objetivos estratégicos políticas de Estado para a melhoria da qualidade da educação básica, valorização e qualificação dos profissionais da educação.

Em consoante com os objetivos estratégicos, avalia-se que problematizar conteúdos, estratégias de ensino, currículo escolar e formação docente poderá ser importante para o enfrentamento do insucesso educacional e a decorrente desigualdade social. Dessa forma, o CT se apresenta igualmente como um objeto de estudo oportuno, uma vez que já se encontra inserido na Base Nacional Comum Curricular brasileira BNCC (BRASIL, 2020) -e, também, na Resolução CNE/CP nº 2 (BRASIL, 2019), que define as Diretrizes Curriculares Nacionais para a Formação Inicial de Professores para a Educação Básica e institui a Base Nacional Comum para a Formação Inicial de Professores da Educação Básica (BNC-Formação). Outrossim, ainda que o CT conste nas diretrizes escolares e formativas dos profissionais dos anos iniciais do ensino fundamental da educação básica, como já destacado, é importante mencionar, tanto sua recente inserção nesses documentos, quanto sua trajetória histórica e conceitual.

Brackmann (2017) e Vicari et al. (2018) mencionam a grande repercussão do termo através de Jeannette Wing, em 2006. Wing inicialmente cunhou o termo CT como uma habilidade essencial para qualquer pessoa, independentemente de sua área de formação ou atuação. Após algumas reformulações conceituais a pesquisadora passou a explicar o CT como a organização dos esquemas mentais necessários para a formulação ou solução de problemas, sob a forma mais eficaz, passíveis de realização por uma pessoa ou por uma máquina. No entanto, com base na exploração prévia da literatura a respeito do CT, parece unânime a dificuldade na obtenção de consenso a respeito de um termo conceitual e estratégia de emprego didático-pedagógico mais apropriada para esse objeto de estudo. Valente (2016), mencionando igualmente essa dificuldade, destaca que a International Society for Technology in Education (ISTE) e a American Computer Science Teachers Association (CSTA) propuseram uma definição para norteamento das atividades realizadas na educação básica, a partir dos conceitos de coleta, análise e representação de dados, decomposição de problema, abstração, algoritmos, automação, paralelização e simulação, sem limitações de áreas, podendo ser praticadas e desenvolvidas em todas as disciplinas:

(...) pensamento computacional é um processo de resolução de problemas que inclui (não somente) as seguintes características: formulação de problemas de forma que computadores e outras ferramentas possam ajudar a resolvê-los; organização lógica e análise de dados; representação de dados por meio de abstrações como modelos e simulações; automatização de soluções a partir do pensamento algorítmico; identificação, análise e implementação de soluções visando a combinação mais eficiente e eficaz de etapas e recursos; eneralização e transferência de soluções para uma ampla gama de problemas. (ISTE-CSTA, 2011, p. 7)

Acredita-se então que o CT, se agregado a conteúdos escolares e intencionalmente empregado em estratégias de ensino, pode contribuir na potencialização do desenvolvimento cognitivo de alunos dos anos iniciais do ensino fundamental, uma vez que demanda vários níveis de abstração. Contudo, é igualmente necessário identificar V. $19 \mathrm{~N}^{\mathrm{o}} 1$, julho, 2021 
como os profissionais que irão inseri-lo nos planejamentos didáticos e pedagógicos o percebem. Partindo-se da premissa que o profissional, para fazer uso intencional de uma ferramenta, necessita saber a respeito de sua constituição, sua potencialidade, atividades onde sejam oportunas e possibilidades para desenvolvimento de trabalhos pedagógicos, crê-se que o CT, ao já constituir demanda de avaliação nacional, deve ser melhor explorado.

Valente (2016) e Vicari et. al. (2018) também destacam mudanças ocorridas no currículo de vários países, inclusa a educação básica, através da inserção de conceitos da ciência da computação, tendo em vista a necessidade de desenvolvimento de habilidades do pensamento crítico e computacional, por viabilizarem a formação de produtores de tecnologia digital, contrariamente a meros consumidores. Por outro lado, salientam a atuação de alguns países em uma perspectiva mais ampla, almejando a exploração de conceitos computacionais para a promoção do desenvolvimento do CT. Nesse sentido, Valente (2016) destaca a aparente consonância brasileira.

Desta forma, com vistas à potencialização das estratégias de ensino para uma melhor aprendizagem, pensa-se que é necessário que os docentes tenham conhecimento a respeito do CT, avaliem sua funcionalidade e o utilizem intencionalmente para um melhor desenvolvimento dos processos de ensino e aprendizagem. Isto posto, a revisão de literatura da qual trata este artigo pretende identificar a presença, as formas de abordagem e objetivo de emprego do CT na educação básica, sobretudo, nos anos iniciais, sob a ótica da formação inicial de pedagogos, que são os profissionais habilitados para essa etapa escolar no contexto brasileiro. Na seção a seguir será explicitado o material e o método de análise empregado. Os resultados e discussões sobre os achados sobre o CT na formação inicial de pedagogos e práticas didático-pedagógicas serão apresentados e discutidos na seção 3. As considerações finais encerram este artigo.

\section{Materiais e Método}

Esta pesquisa constitui-se de uma revisão da literatura a respeito do CT junto à formação inicial de pedagogos, ou seja, a formação de professores atuantes nas etapas iniciais da educação básica brasileira. Essa revisão apresenta elementos de um Mapeamento Sistemático, o qual, para Demerval et al. (2017), constitui-se de um modo qualificado, objetivo e sistemático, para confecção do estado da arte de um determinado objeto de pesquisa. Também chamado de revisão de escopo, pretende uma visão mais ampla desse objeto, sobretudo, quando da produção científica ainda incipiente sobre a temática. A Figura 1 ilustra as etapas da pesquisa realizada:
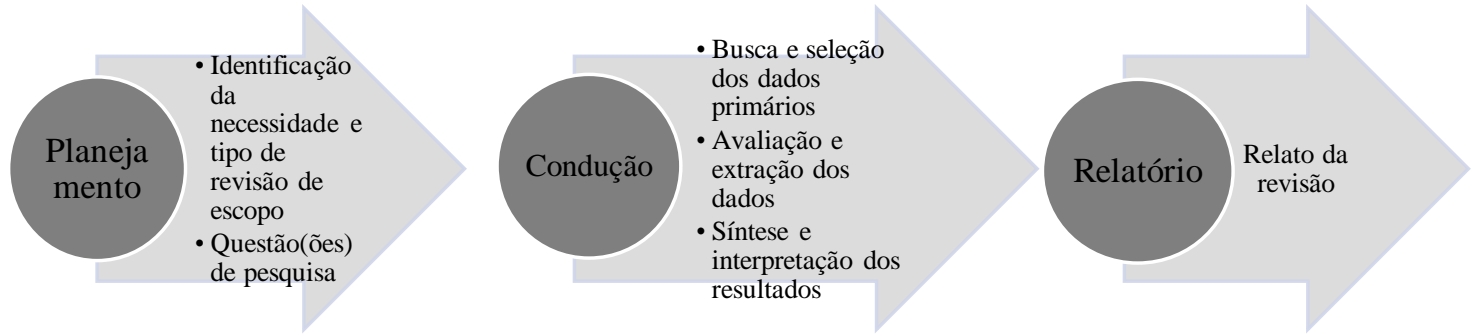

Figura 1 - Adaptação do mapeamento sistemático da literatura com base em Dermeval et al. (2017)

Fonte: Elaborada pelos autores, 2021

$\mathrm{Na}$ etapa de planejamento, além da definição do tipo de revisão bibliográfica a ser realizada, determinou-se as questões norteadoras da pesquisa, a saber: o CT é abordado na formação de professores da educação básica? Em caso afirmativo, de que forma ele é abordado e com que objetivo? 
Na sequência, quanto à parte inicial de condução da pesquisa, a busca por dados primários foi organizada a partir da estratégia de adoção de mecanismo de busca com os termos: "pensamento computacional"; "pensamento computacional e formação de professores" e "formação de professores e educação básica", nos idiomas português, inglês e espanhol. As bases de dados percorridas foram o Lume, área de teses e dissertações, SciELO e o Portal de Periódicos da CAPES. Adotou-se como critérios de exclusão dos textos científicos aqueles que não tratassem sobre o ensino, formação ou aplicação do pensamento computacional em atividades com discentes da educação básica, ou ao respectivo corpo docente. O período das ocorrências também foi limitado aos anos entre 2015 e 2020 (ano em que a pesquisa foi realizada)

\section{Resultados e Discussões}

De acordo com as delimitações estabelecidas, foram selecionadas 06 ocorrências no Lume (teses e dissertações), 09 no SciELO e 12 no portal de periódicos da CAPES, ou seja, 27 ocorrências potenciais (Quadro1). A tabela 1 relaciona os mecanismos de busca e bases de dados, o número de ocorrências e os resultados selecionados.

Tabela 1 - Bases de dados e delimitações da pesquisa

\begin{tabular}{l|c|c|c|c|c|c}
\hline & \multicolumn{5}{c}{ TERMOS DE BUSCA } \\
\hline $\begin{array}{l}\text { BASE DE } \\
\text { DADOS }\end{array}$ & \multicolumn{2}{|l|}{$\begin{array}{l}\text { Pensamento } \\
\text { Computacional }\end{array}$} & $\begin{array}{l}\text { Pensamento Computacional e } \\
\text { formação de professores }\end{array}$ & $\begin{array}{l}\text { Formação de } \\
\text { professores e educação } \\
\text { básica }\end{array}$ \\
\cline { 2 - 7 } & Retorno & Selecionados & Retorno & Selecionados & Retorno & Selecionados \\
\hline LUME & 04 & 04 & 00 & 00 & 18 & 02 \\
\hline SciELO & 20 & 06 & 02 & 01 & $23^{1}$ & 02 \\
\hline $\begin{array}{l}\text { Portal de } \\
\text { periódicos da } \\
\text { CAPES }\end{array}$ & 17.764 & 00 & 1.409 & 04 & 1.561 & 08 \\
\hline
\end{tabular}

Fonte: Elaborada pelos autores, 2021

Quadro 1 - Resultados selecionados a partir das bases de dados consultadas

\begin{tabular}{|l|l|}
\hline BASE DE DADOS & \multicolumn{1}{c|}{ REFERENCIA } \\
\hline LUME & $\begin{array}{l}\text { Brackmann (2017); Boucinha (2017); Castilho (2018); Morais (2016); Fontes } \\
\text { (2019) e Longo (2019) }\end{array}$ \\
\hline SciELO & $\begin{array}{l}\text { Morais, Basso, Azevedo e Fagundes (2017); Chimunja, Collazos e Hurtado } \\
\text { (2017); Dapozo, Petris, Greiner, Espíndola, Company e López (2016); } \\
\text { Fracchia, Kogan e Amaro (2016); Torres, González e Carvalho (2018); Redón } \\
\text { e Aroca (2018); Piqueira (2016); Monjelat (2019) e Santos, Almeida e } \\
\text { Zanotello (2018) }\end{array}$ \\
\hline $\begin{array}{l}\text { Portal de periódicos } \\
\text { da CAPES }\end{array}$ & $\begin{array}{l}\text { Santos, Soares, Bianco, Filho e Lahm (2016); Valente (2016); Gonçalves, } \\
\text { Portella e Luz (2019); Paz (2017); Howard (2019); Dinçer (2018); Adler e } \\
\text { Kim (2018); Jaipal-Jamani, Kamini e Angeli, (2017); Strawhacker, Lee e Bers } \\
\text { (2018); Ronsivalle, Boldi, Gusella, Inama e Carta (2019); Erstad, Eickelmann } \\
\text { e Eichhorn (2015) e Zhong, Wang, Chen e Li (2017) }\end{array}$ \\
\hline
\end{tabular}

Fonte: Elaborado pelos autores, 2021

\footnotetext{
${ }^{1}$ Dado o elevado número de ocorrências de trabalhos, optou-se pela associação do termo "tecnologia", a fim de restringir o número de retornos mais adequados ao tema de estudo.

${ }^{2}$ Frente ao número elevado de ocorrências, exclusivamente nessa base de dados, houve a necessidade de um maior refino nos termos de busca, sendo então limitada às ocorrências inclusas nos tópicos Educational technology; Teaching Methods

V. $19 \mathrm{~N}^{\circ} 1$, julho, 2021

DOI: https://doi.org/10.22456/1679-1916.118416 RENOTE
} 
O cenário da educação básica apresentado pelas obras é, de certa forma, sintetizado por Brackmann (2017), ao mencionar o descompasso entre a escola, computadores e seus usos, o que faria emergir a necessidade de novas competências. Erstad et al. (2015), Valente (2016) e Strawhacker et al. (2018) vão além, afirmando que, embora o acesso e disponibilidade de tecnologias nos ambientes escolares tenha melhorado, ainda existem obstáculos, como crenças, atitudes, ceticismo sobre integração tecnológica, além de questões quanto à avaliação da aprendizagem e treinamento adequado dos professores. Esses autores também discutem a frequente crítica aos professores por estes serem relutantes às mudanças, sobretudo, quanto ao uso das tecnologias da informação e comunicação nos processos de ensino e aprendizagem. Em contraponto, apontam os professores como os principais agentes de mudança desse cenário, mencionando-os como parte de diferentes iniciativas em todas as esferas governamentais. Convergente aos quatro autores está a preocupação de formação docente adequada para a era digital, sendo destacada a necessidade de estratégias vinculadas à prática, política e pesquisa. Para os autores, a tensão entre estratégias docentes antigas e novas é um desafio que precisa ser enfrentado agora, sendo necessário tornar as estruturas contemporâneas e a pesquisa sobre competências do século XXI mais acessíveis aos professores em suas práticas. Igualmente necessário é o envolvimento por parte dos professores na discussão do futuro da educação e sua transformação em agentes de mudança com uso de novas tecnologias.

Dapozo et al. (2016) e Longo (2019) abordam uma investigação diagnóstica das competências dos professores de anos iniciais do ensino básico em suas aulas de matemática. Dapozo et al. (2016), porém, com foco sobre seus conhecimentos a respeito de recursos tecnológicos e implementação em sala de aula, obteve dados que ratificam a necessidade de uma formação adequada. Os resultados apontaram que muitos docentes não desenvolvem as competências básicas para o uso de tecnologias de informação e comunicação, seja em sua formação inicial, quanto na continuada, fato que não colabora para a potencialização das aprendizagens de seus alunos em sala de aula. Ambos os autores consideram importante a afinidade dos docentes com a matemática e com a educação, uma vez que a formação de sujeitos com uma estrutura de pensamento ordenada e lógica auxilia no enfrentamento de problemas e a viverem melhor. Longo (2019) destaca, ainda, que embora sejam feitos esforços através de pesquisadores, na tentativa de desnaturalizar práticas obsoletas na matemática, as práticas naturalizadas se impõem, contrariando possibilidades de produção de conhecimento em diferentes contextos, dentro da prática social, valorizando diferentes formas de pensar e as atuais características tecnológicas dos sujeitos. Também são mencionadas deficiências nos currículos dos cursos de formação docente, com escassa inserção sobre o ensino e aprendizagem de matemática.

Como percebemos, é frequente a observância sobre a carência nas formações iniciais que capacitem docentes para potencialização dos distintos espaços e formas de aprendizagem, assim como a crítica aos currículos, que não se apresentam como suficientes para o ensino de matemática ou uso de recursos tecnológicos, a exemplo das demais obras consultadas em paralelo. Dinçer (2018), semelhante aos demais, também foca seu estudo na formação docente, porém, restrito aos professores de educação infantil e no nível de alfabetização tecnológica do grupo, ou seja, avaliando o conhecimento, as habilidades e as atitudes, pois tratando-se de conceitos diferentes, precisariam ser examinados em conjunto para determinar o nível de alfabetização. Os dados, análogos ao de Dapozo et al. (2016), revelaram que os professores não eram alfabetizados em tecnologia o suficiente, apesar de afirmarem o contrário, indicativo que impactaria diretamente em suas práticas profissionais. $\mathrm{O}$ fato de os professores não participarem de 
nenhum curso relacionado à tecnologia em seus programas de formação de professores ou de terem cursos com conteúdo insuficiente foi considerado um dos principais motivos desse baixo nível. Assim, o autor sugere que os cursos sobre alfabetização tecnológica devam ser suficientemente ministrados na formação de professores. Fato que também manterá relação direta com suas crenças e habilidades tecnológicas.

Retomando a questão das crenças, ou seja, da cultura docente, Howard (2019) nos auxilia para a percepção de sua importância, ao tratar de relatos de iniciativas de implementação de ensino onde estavam presentes a codificação, programação de computadores e o CT, com vistas à obtenção de uma vantagem competitiva futura dos alunos, e facilitação no aprendizado de outras disciplinas do Ensino Básico. Segundo o autor, frente à incipiência de uma metodologia de ensino, formação e cultura docente relacionada à Ciência da Computação, assim como no que diz respeito à pesquisa relacionada a sua implementação em escolas de educação básica, o conhecimento prévio sobre as crenças docentes dos formadores em relação ao tema ajudaria a entender eventuais obstáculos iniciais com os docentes escolares.

Especificamente no que se refere ao CT, similar à Howard (2019), Brackmann (2017), Boucinha (2017), Chimunja et al. (2017) e Monjelat (2019) mencionam a falta de consenso internacional a respeito do conceito de CT, assim como a dificuldade no estabelecimento de uma metodologia para seu emprego, especialmente com crianças, e formas de avaliação dos resultados. Já Paz (2017) problematiza e qualifica os processos de ensino e aprendizagem, ao questionar a formação de professores em razão da necessidade de novas competências para ensinar, destacando o CT para auxílio nos processos envolvidos na formulação dos problemas reais e nas suas soluções. Ressalta a importância no foco da qualidade das formações ofertadas, contrariando a tendência na preocupação apenas quantitativa de profissionais, sem vínculo com o projeto político pedagógico da escola. Defende uma formação com exploração colaborativa, onde os pares troquem ideias, sugestões a partir de suas realidades escolares, lembrando também princípios da andragogia. Segundo a autora, são equivocados os cursos que definem a tecnologia como um fim, e não como meio de apoio à pedagogia.

Assim, dada a emergência de desenvolvimento de novas habilidades e competências no século XXI, Brackmann (2017) aponta um encadeamento de ações relativas à promoção do CT: conscientização dos legisladores a respeito da distinção entre a computação (físico e lógico) da informática (TICs - Tecnologias da Informação e Comunicação), uma vez que os princípios da computação são importantes, devendo ser ensinados desde o ensino básico, havendo, inclusive, apontamento científicos a respeito dessa introdução; desenvolvimento de material pedagógico; formação docente, sobretudo, para cursos restritos à área da educação; oferta de disciplinas eletivas e, a exemplo de outros governos, iniciativas de competições envolvendo a computação na solução de problemas, com vistas ao envolvimento dos estudantes com o tema.

Sob o aspecto quantitativo dos dados em relação às ocorrências na educação básica proporcionado por essa pesquisa, pode-se mencionar que, no que diz respeito à formação docente, Dinçer (2018) e Santos et al. (2016) voltam-se apenas para a etapa da educação infantil. Já Strawhacker (2018), centra-se em parte da educação infantil e anos iniciais. Longo (2019), Dapozo et al. (2016), restritamente para os anos iniciais. Monjelat (2019), Jaipal-Jamani et al. (2017) e Ronsivalle et al. (2019) dedicaram-se à formação de professores para toda a etapa do ensino fundamental. Já Adler e Kim (2018), ensino fundamental e médio. Fontes (2019), Gonçalves et al. (2019), Howard (2019), Morais (2017), Valente (2016), Erstad et al. (2015) e Paz (2017) produzem sob um âmbito mais generalista de formação, não se limitando, necessariamente, a uma das etapas da educação 
básica. Logo, pode-se perceber que, quantitativamente, as atividades de formação docente estão mais voltadas para a etapa do ensino fundamental, sobretudo, para os anos finais.

Especificamente quanto à abordagem do CT através de atividades pedagógicas com discentes na educação básica, na educação infantil, apenas Torres et al. (2018) narra uma proposta didática piloto e Santos et al. (2018) nos anos iniciais. Já Brackmann (2017) e Chimunja et al. (2017) mesclam alunos do último ano dos anos iniciais e finais. Boucinha (2017) e Zhong et al. (2017) se limitam a alunos de anos finais do ensino fundamental. Em nova mescla, Morais (2018) com alunos de anos finais e ensino médio. Já Castilho (2018), Fracchia et al. (2016) e Redón e Aroca (2018) dedicaram-se apenas a experiências com o ensino médio. Por fim, Piqueira (2016), apresenta conceitos de complexidade computacional algorítmica e informacional, não sendo relatadas experiências formativas ou pedagógicas. Nota-se, mais uma vez, que a educação infantil e os anos iniciais são as etapas com menor incidência de resultados, elevando-se o número relativo às ocorrências no ensino médio.

Sob a ótica da forma de abordagem do CT, percebeu-se que a maior incidência pauta o uso da linguagem de programação, com dez ocorrências, seguido da robótica, com quatro ocorrências. Estes resultados parecem também ratificar a baixa exploração do CT na educação infantil e anos iniciais do ensino fundamental, uma vez que seus empregos exigem certo nível de organização cognitiva. A alfabetização tecnológica foi mencionada em três ocorrências. Já as atividades desplugadas encontram-se em número similar ao emprego de recursos tecnológicos, que somam duas ocorrências. Apenas uma ocorrência menciona a abordagem através de teste e bateria de provas de raciocínio.

Os objetivos quanto à abordagem do CT na educação básica parecem conciliar o uso da programação como estratégia didática e promotora de habilidades sociais em três dimensões: cognitiva, ágil e colaborativa, como reportam as nove ocorrências. Importante destacar que o uso foi relatado tanto para formação docente quanto em atividades pedagógicas discentes. A seguir, o objetivo de emprego do CT como facilitador para aprendizagem em disciplinas diversas aparece em quatro ocorrências, similarmente ao número de emprego com o objetivo de desenvolvimento do raciocínio lógico. Nos demais trabalhos, são encontradas experiências para a verificação dos resultados com o emprego de computação desplugada ou discussões referentes às necessidades contemporâneas para a adequada formação docente.

Percebeu-se, sob um aspecto geral, que a temática do CT na educação básica, embora seja tida como competência fundamental para todos os sujeitos, ainda necessita avançar sobre o aspecto de certa uniformização conceitual, metodológica e avaliativa para o ensino, além da formativa e cultural para o nível docente. Seus conceitos relacionamse a elementos da ciência da computação e parecem estar, analogamente ao uso de recursos tecnológicos, ainda incipientes na cultura educacional da educação básica. Quantitativamente, os dados revelam, além de um maior número de experiências formativas com docentes em relação a discentes, uma centralidade sob o uso da linguagem de programação, como estratégia didática, no intuito da promoção do desenvolvimento cognitivo, seguido do aspecto atitudinal e de colaboração. Em paralelo, também foi percebida uma menor incidência de explorações, sejam docentes ou discentes, junto à etapa da educação infantil e anos iniciais do ensino fundamental da educação básica.

\section{Considerações Finais}

A revisão de literatura auxiliou na elucidação de elementos importantes para o desenvolvimento e reflexão sobre o CT na educação básica. Sobre as questões de pesquisa inicialmente elaboradas chegamos às seguintes conclusões: 
1) Compreendeu-se que o CT está elencado como uma das novas competências emergentes e necessárias para a formação e desenvolvimento dos sujeitos do século XXI nas pesquisas apreciadas. De acordo com o número de ocorrências, também se evidenciou o problema da formação docente, ainda incipiente na área, assim como a baixa produção de metodologias e práticas didático-pedagógicas em todas as etapas da educação básica.

2) As experiências de implementação do CT parecem estar centradas no ensino de linguagem de programação ou fortemente ligadas ao ensino de matemática. Contudo, há algumas experiências com robótica e atividades desplugadas.

3) Percebeu-se, também, ocorrências de estudo a respeito das contribuições do CT, elencando o desenvolvimento cognitivo como importante componente, seguido do aspecto atitudinal e de colaboração.

Ainda com base nos autores, tem-se que o CT está em vias de implementação nos currículos escolares da educação básica em vários países, justamente pela visão do possível impacto positivo de desenvolvimento dos alunos mais precocemente. Contudo, ainda há controvérsias a respeito de sua abordagem, ou seja, se restrito à programação ou sob uma visão mais ampla, aplicável a qualquer conteúdo escolar e vivência prática, fato que parece estar intimamente ligado às divergências conceituais a seu respeito.

Logo, ao pensarmos em iniciativas de inserção do CT no contexto educacional brasileiro, pensa-se que, apesar de já haver amparo para seu desenvolvimento em documentos educacionais oficiais e norteadores, uma maior solidez formativa, tecnológica e cultural no âmbito docente se faz necessária, o que torna o CT ainda mais desafiador e demandante de contribuições de pesquisas científicas, sobretudo, para a educação básica.

\section{Referências}

Adler, R; Kim, H. Enhancing future K-8 teachers' computational thinking skills through modeling and simulations. Education and Information Technologies, 2018, Vol.23(4), pp.1501-1514. Disponível em: < https://link-springer-

com.ez45.periodicos.capes.gov.br/article/10.1007/s10639-017-9675-1>. Acesso em Abr. 2020.

Boucinha, R. M. Aprendizagem do pensamento computacional e desenvolvimento do raciocínio. Porto Alegre: PPGIE/UFRGS, 2017. 151p. Tese de Doutorado.

Brackmann, C. P. Desenvolvimento do pensamento computacional através de atividades desplugadas na educação básica. Porto Alegre: PPGIE/UFRGS, 2017. Tese de Doutorado.

Brasil, Ministério da Educação. Compromisso Nacional pela Educação Básica. Disponível em: < http://portal.mec.gov.br/images/11.07.2019_Apresentacao-edbasica.pdf > Acesso em: Mar. 2020.

Brasil. Ministério da Educação. Base Nacional Comum Curricular. Disponível em: $<$ http://portal.mec.gov.br/index.php?option $=$ com_docman\&view=download\&alias=796 11-anexo-texto-bncc-aprovado-em-15-12-17-pdf\&category_slug=dezembro-2017pdf\&Itemid=30192> Acesso em Mar. 2020.

Brasil, Ministério da Educação. Define as Diretrizes Curriculares Nacionais para a Formação Inicial de Professores para a Educação Básica e institui a Base Nacional Comum para a Formação Inicial de Professores da Educação Básica (BNC- 
Formação). Resolução CNE/CP nº 2/2019. Disponível em:

<http://portal.mec.gov.br/component/content/article?id=12991> Acesso em Nov. 2020.

Castilho, M. I. Hiperobjetos da robótica educacional como ferramentas para o desenvolvimento da abstração reflexionante e do pensamento computacional. Porto Alegre: PPGIE/UFRGS, 2018. Tese de Doutorado.

Chimunja, A. M.; Collazos, C. A.; Hurtado, J. A. ChildProgramming-C: como una mejora de la dimensión colaborativa del modelo ChildProgramming. Entre Ciencia e Ingeniería, Dez 2017, Vol. 11, № 22, pp 67 - 75. Disponível em: http://www.scielo.org.co/scielo.php?script=sci_arttext\&pid=S190983672017000200067\&lang=pt> Acesso em Jan. 2020.

Dapozo, G.; Petris, R.; Greiner, C.; Espíndola, M. C.; Company, A.M.; López, M. Capacitación en programación para incorporar el pensamiento computacional en las escuelas. Revista Iberoamericana de Tecnología en Educación y Educación en Tecnología, Dez 2016, № 18, pp 113 - 121. Disponível em: < http://www.scielo.org.ar/scielo.php?script=sci_arttext\&pid=S185099592016000200012\&lang=pt> Acesso em Jan. 2020.

Dermeval, D.; Coelho, J.A. P. de M.; Bittencourt, I. I. Mapeamento Sistemático e Revisão Sistemática da Literatura em Informática na Educação. In: Jaques, P. A.; Siqueira; S.; Bittencourt, I; Pimentel, M. (Org.) Metodologia de Pesquisa Científica em Informática na Educação: Abordagem Quantitativa. Porto Alegre: SBC, 2020.

Dinçer, S. Are preservice teachers really literate enough to integrate technology in their classroom practice? Determining the technology literacy level of preservice teachers. Education and Information Technologies, 2018, Vol.23(6), pp.2699-2718. Disponível em: < https://link-springer-

com.ez45.periodicos.capes.gov.br/article/10.1007/s10639-018-9737-z> Acesso em Abr. 2020 .

Erstad, O.; Eickelmann, B.; Eichhorn, K. Preparing teachers for schooling in the digital age: A meta-perspective on existing strategies and future challenges. Education and Information Technologies, 2015, Vol.20(4), pp.641-654. Disponível em: $<$ https://link-springer-com.ez45.periodicos.capes.gov.br/article/10.1007/s10639-0159431-3> Acesso em Abr. 2020.

Fontes, M. G. Programa Nacional de Formação de Professores da Educação Básica: um estudo sobre suas contribuições para a adequação da formação docente para o ensino de ciências e de matemática. Porto Alegre: PPGCQVS/UFRGS, 2019. Dissertação de Mestrado.

Fracchia, C. C.; Kogan, P.; Amaro, S. Competir + Motivar + Hornero = aprender programación. Revista Iberoamericana de Tecnología en Educación y Educación en Tecnología, Dez 2016, № 18, pp 19 - 29. Disponível em: < http://www.scielo.org.ar/scielo.php?script=sci_arttext\&pid=S185099592016000200003\&lang=pt > Acesso em Jan. 2020.

Howard, N. EdTech Leaders' Beliefs: How are K-5 Teachers Supported with the Integration of Computer Science in K-5 Classrooms? Technology, Knowledge and Learning, 2019, Vol.24(2), pp.203-217. Disponível em: < https://link-springercom.ez45.periodicos.capes.gov.br/article/10.1007/s10758-018-9371-2> Acesso em Abr. 2020. 
Jaipal-Jamani, K.; Angeli, C. Effect of Robotics on Elementary Preservice Teachers' Self-Efficacy, Science Learning, and Computational Thinking. Journal of Science Education and Technology, 2017, Vol.26(2), pp.175-192. Disponível em: < https://link-springer-com.ez45.periodicos.capes.gov.br/article/10.1007/s10956-0169663-z> . Acesso em Abr. 2020.

Longo, F. A Docência em Matemática nos Anos Iniciais: enunciados que a constitui. Porto Alegre: PPGEDU/UFRGS, 2019. Dissertação de Mestrado.

Monjelat, N. Programación de tecnologías para la inclusión social con Scratch: Prácticas sobre el pensamiento computacional en la formación docente. Revista Electrónica Educare, Dez 2019, Vol. 23, № 3, pp.182-206. Disponível em: < https://www.scielo.sa.cr/scielo.php?script=sci_arttext\&pid=S1409$42582019000300182 \&$ lang=pt $>$ Acesso em Jan. 2020.

Morais, A. D. de. O desenvolvimento do raciocínio condicional a partir do uso de teste no squeak etoys. Porto Alegre: PPGIE/UFRGS, 2016. Tese de Doutorado.

Morais, A. D. de; Basso, M. V. de A.; Fagundes, L. da C. Educação Matemática \& Ciência da Computação na escola: aprender a programar fomenta a aprendizagem de matemática? Ciência \& Educação (Bauru), Jun 2017, Vol. 23, № 2, pp. 455 - 473. Disponível em : $<$ http://www.scielo.br/scielo.php?script=sci_arttext\&pid=S1516$73132017000200455 \&$ lang=pt $>$ Acesso em Jan 2020.

Paz, L. A. S. do C. O pensamento computacional e a formação continuada de professores: uma experiência com as TICs. Revista on line de Política e Gestão Educacional, Araraquara, p. 1655-1677, 2017. Disponível em: <https://periodicos.fclar.unesp.br/rpge/article/view/10095>. Acesso em 22 jul. 2021.

Piqueira, J. R. C. Complexidade computacional e medida da informação: caminhos de Turing e Shannon. Estudos Avançados, Ago 2016, Vol. 30, № 87, pp. 339- 344. Disponível em: < http://www.scielo.br/scielo.php?script=sci_arttext\&pid=S010340142016000200339\&lang=pt > Acesso em Jan. 2020.

Redón, Á. B.; Aroca, Á. S. Aprendizaje de las matemáticas a través del lenguaje de programación $R$ en Educación Secundaria. Educación matemática, 2018, Vol. 30, Nº 1, pp. 133-162 Disponível em < http://www.scielo.org.mx/scielo.php?script=sci_arttext\&pid=S166558262018000100133\&lang=pt > Acesso em Jan. 2020.

Ronsivalle, G.; Boldi, A.; Gusella, V.; Inama, C.; Carta, S. How to Implement Educational Robotics' Programs in Italian Schools: A Brief Guideline According to an Instructional Design Point of View. Technology, Knowledge and Learning, 2019, Vol.24(2), pp. 227-245. Disponível em: < https://link-springercom.ez45.periodicos.capes.gov.br/article/10.1007/s10758-018-9389-5> Acesso em Abr. 2020 .

Santos, E. R. S.; Soares, G.; Bianco, G. D.; Filho, J. B. R.; Lahm, R. A. Estímulo ao Pensamento Computacional a partir da Computação Desplugada: uma proposta para Educação Infantil. Revista Latinoamericana de Tecnología Educativa, 2016, V. 15, N. 3, pp. 99-112. Disponível em: < https://mascvuex.unex.es/revistas/index.php> Acesso em Jan. 2020.

Santos, V. G.; Almeida, S. E. de; Zanotello, M. A sala de aula como um ambiente equipado tecnologicamente: reflexões sobre formação docente, ensino e aprendizagem nas séries iniciais da educação básica. Revista Brasileira de Estudos V. $19 \mathrm{~N}^{\mathrm{o}} 1$, julho, 2021 RENOTE DOI: https://doi.org/10.22456/1679-1916.118416 
Pedagógicos, Ago 2018, Volume 99, N²52, pp. 331-349. Disponível em: < http://www.scielo.br/scielo.php?script=sci_arttext\&pid=S217666812018000200331\&lang=pt> Acesso em Jan. 2020.

Strawhacker, A.; Lee, M.; Bers, M. Teaching tools, teachers' rules: exploring the impact of teaching styles on young children's programming knowledge in ScratchJr. International Journal of Technology and Design Education, 2018, Vol.28(2), pp.347-

376. Disponível em: < http://web-a-

ebscohost.ez45.periodicos.capes.gov.br/ehost/detail/detail?vid=0\&sid=0fb33e90-b921$4 \mathrm{ed} 8-95 \mathrm{e} 8$ -

3088164b0b0d\%40sessionmgr4007\&bdata=Jmxhbmc9cHQtYnImc210ZT1laG9zdC1sa XZl\#AN=129629813\&db=aph> Acesso em Abr. 2020.

Torres, N. B.; González, R. L.; Carvalho, J. L. Roamer, un robot en el aula de Educación Infantil para el desarrollo de nociones espaciales básicas. RISTI Revista Ibérica de Sistemas e Tecnologias de Informação, Set 2018, N 28, pp. 14 - 28 Disponível em: < http://www.scielo.mec.pt/scielo.php?script=sci_arttext\&pid=S164698952018000300003\&lang=pt > Acesso em Jan. 2020.

Valente, J. A. Integração do pensamento computacional no currículo da educação básica: Diferentes estratégias usadas e questões de formação de professores e avaliação do aluno. Revista e-Curriculum, São Paulo, 2016, v. 14, n. 3, pp. 864-897. Disponível em: < http://revistas.pucsp.br/index.php/curriculum> Acesso em Jan. 2020.

Vicari, R. M. Pensamento computacional: revisão bibliográfica. Porto Alegre: UFRGS/MEC, 2018. 192p. Disponível em: < http://www.bibliotecadigital.ufrgs.br/da.php?nrb=001097710\&loc=2019\&l=2c3152e31f 6ea69e> Acesso em Abr. 2020.

Zhong, B.; Wang, Q.; Chen, J.; Li, Y. Investigating the period of switching roles in pair programming in a primary school. Educational Technology \& Society, 2017, Vol.20(3), p.220(14). Disponível em: < https://drive.google.com/file/d/1945EjnWRPNPs2plCZxomiqNSYuVdUhuH/view> Acesso em Jan. 2020. 